\title{
Banach lattice algebras: some questions, but very few answers
}

\author{
A. W. Wickstead ${ }^{1}$
}

Received: 10 September 2015 / Accepted: 17 November 2015 / Published online: 11 December 2015 C) The Author(s) 2015. This article is published with open access at Springerlink.com

\begin{abstract}
We pose a number of questions and problems about Banach lattice algebras. These concern: What should the definition be? How to add an identity. Order theoretic properties of the multiplication. Order theoretic properties of the left regular representation.
\end{abstract}

Keywords Riesz algebras · Representations $\cdot$ Norms $\cdot$ Banach Lattice algebras

Mathematics Subject Classification 06A70

\section{Introduction}

As part of the Ordered Banach Algebras workshop that was held at the Lorentz Center in Leiden during the week 21-25 July, 2014 I gave a presentation on Banach lattice algebras. My motivation was the realization that although there are scattered results in the literature that refer to Banach lattice algebras, there has been no systematic study of them and indeed the literature is not even consistent as to what one of these objects is! The results in the literature that I am aware of seem to concentrate either on spectral properties, usually with a view to answering questions about operators on Banach lattices, or to characterising some of the concrete examples mentioned below. I hoped with my presentation, and in this article, to point out the wealth of rather fundamental open problems that are out there, at least some of which should be fairly elementary for someone with the right background. In order to avoid undue repetition, I will not recap the basic theory either of Banach algebras, which may be found in

$\triangle$ A. W. Wickstead

a.wickstead@qub.ac.uk

1 Pure Mathematics Research Centre, Queen's University Belfast, Belfast BT7 1NN, Ireland, UK 
$[2,9,15,24,25,35]$ amongst other places, or of Banach lattices, for which I refer the reader to $[1,23,26,33,34]$.

What is worth pointing out at this stage is the wealth of examples of objects which possess both of these structures in a natural way. In the following examples, I will be deliberately vague as to what scalar field is involved.

Example 1.1 (1) If $\Sigma$ is a locally compact Hausdorff space then $C_{0}(\Sigma)$, the continuous scalar functions which vanish at infinity, is a Banach space under the supremum norm. It is a Banach algebra under the pointwise multiplication

$$
(f \star g)(\sigma)=f(\sigma) g(\sigma)
$$

and if $\Sigma$ is compact then the identity (the constantly one function) has norm one. For the pointwise order,

$$
f \geq g \Leftrightarrow f(\sigma) \geq g(\sigma) \quad \forall \sigma \in \Sigma,
$$

it is a Banach lattice. It is also clear that if $f, g \geq 0$ then $f \star g \geq 0$.

(2) A topological group $G$ is a Hausdorff space which is also a group with the map $(g, h) \mapsto g h^{-1}: G \times G \rightarrow G$ being continuous. If $G$ is locally compact then it is automatically completely regular so there are many continuous real-valued functions on it. If $G$ is locally compact there is always a left invariant Haar measure $\mu$ on $G$, i.e. $\mu(g A)=\mu(A)$ for all $g \in G$ and all Borel subsets $A$ of $G$, where $g A=\{g a: a \in A\}$. Such a $\mu$ is unique to within a positive multiplicative constant. If $G$ is compact it is usual to choose $\mu(G)=1$. If $G$ is either compact or abelian then $\mu$ is also right invariant, i.e. $\mu(A g)=\mu(A)$ for all $g \in G$ and all Borel subsets $A$ of $G$, but that is not true in general. If $\phi$ is a real-valued function on $G$ then I will write $\int \phi(g) d g$ for the integral of $\phi$ with respect to Haar measure. The Banach space $L^{1}(G)$ is normed by $\|\phi\|_{1}=\int|\phi(g)| d g$. If we define $\phi \geq \psi$ to mean that $\phi(g) \geq \psi(g)$ ( $\mu$-almost everywhere) then $L^{1}(G)$ becomes a Banach lattice. The convolution multiplication on $L^{1}(G)$ is defined by

$$
(\phi \star \psi)(g)=\int \phi(g h) \psi\left(h^{-1}\right) d h
$$

and this makes $L^{1}(G)$ into a Banach algebra. $L^{1}(G)$ is commutative if and only if the group $G$ is abelian. $L^{1}(G)$ has a multiplicative identity if and only if $G$ is discrete, in which case the identity certainly has norm equal to one. Again, it is clear that if $\phi, \psi \geq 0$ then $\phi \star \psi \geq 0$.

(3) The space of bounded regular Borel measures on a locally compact topological group $G, M(G)$, with norm $\|\mu\|$ being the total variation of $\mu$, is a Banach space. With the order $\mu \geq v$ meaning that $\mu(E) \geq v(E)$ for all Borel sets $E$, it is a Banach lattice. The convolution multiplication defined for Borel sets $E$ by

$$
(\mu \star v)(E)=(\mu \times v)(\{(g, h) \in G \times G: g h \in E\})
$$

makes it into a Banach algebra. Again, it is clear that if $\mu, v \geq 0$ then $\mu \star v \geq 0$. 
(4) If $E$ is a Banach lattice then a linear operator $T: E \rightarrow E$ is positive if $x \geq$ $0 \Rightarrow T x \geq 0$. Positive operators are automatically bounded for the usual operator norm. The linear span of the positive operators is the space of regular operators, denoted by $\mathcal{L}^{r}(E)$. It is rare that this is the same as $\mathcal{L}(E)$. Two important cases where it holds are if $E=C(\Omega)$, where $\Omega$ is a Stonean space, i.e. $\Omega$ is a compact Hausdorff space in which the closure of every open set is open, or if $E$ is an L-space. The first of these cases dates back to [20] whilst the second is in [21]. In general, $\mathcal{L}^{r}(E)$ is not complete for the operator norm. We define $S \geq T$ to mean that $S-T$ is a positive operator. In general $\mathcal{L}^{r}(E)$ is not a lattice under this order. The regular norm (or $r$-norm) on $\mathcal{L}^{r}(E)$ is

$$
\|T\|_{r}=\inf \{\|S\|: S \geq T,-T\} .
$$

$\mathcal{L}^{r}(E)$ is complete under the regular norm. If $E$ is Dedekind complete, i.e. if every non-empty set that is bounded above has a supremum, then $\mathcal{L}^{r}(E)$ with the regular norm is actually a Banach lattice and the norm may be more simply described by $\|T\|_{r}=\||T|\|$. The composition of two positive operators is certainly positive so that $\mathcal{L}^{r}(E)$ is closed under composition. This makes $\mathcal{L}^{r}(E)$ with the regular norm into a Banach algebra, and the identity has norm one.

All of these examples share the features of being at the same time both a Banach algebra and a Banach lattice with at least the minimum connection between the two structures that the product of positive elements is positive. Schaefer phrases this in a more suggestive manner that is easily seen, at least in the real case, to be equivalent, namely that (writing $\star$ for the multiplication) $|x \star y| \leq|x| \star|y|$ for all $x, y$. If we were to ignore the norm, this is saying that we have a Riesz algebra.

The left regular representation of a Banach algebra $A$ is the mapping $\pi: a \mapsto L_{a}$ where $L_{a}$ is the bounded linear operator on $A$ defined by $L_{a}(x)=a \star x$. This is always a multiplication preserving map of $A$ into $\mathcal{L}(A)$, the algebra of bounded operators on $A$ under composition. If $A$ has an identity then $\pi$ is a unital algebra isomorphism, and if we assume that the identity has norm one then it is an isometry. So the study of Banach algebras with an identity of norm one is just the study of closed unital subalgebras of the bounded operators on a Banach space.

In the remainder of this paper, I will pose explicit questions and problems where possible, but much of the discussion will (of necessity at this stage) be rather vague and not such as to admit of explicit questions. The reader who really wants to work in this area should be aware that some suggestions for further research are embedded in the text rather than explicitly displayed as questions.

\section{What is a Banach lattice algebra?}

From the literature on Banach lattice algebras (and that on ordered Banach algebras) there does not appear to be a consensus on a definition.

What is agreed is that one should be a Banach lattice, be an associative algebra with a sub-multiplicative norm and that the product of positive elements should be positive. 
Where authors differ is on the matter of the multiplicative identity, if there is one. Must it be positive? Must it have norm equal to one? Scheffold, in an unpublished result, showed that if an identity has norm one then it must be positive. A nice proof of this may be found in [7]. A Banach algebra with an identity that does not have norm one can always be re-normed so that the identity does have norm one. That is not possible here as this re-norming may destroy the lattice property of the norm. To cover as wide a range of examples as possible and in an effort to standardise terminology we propose that a Banach lattice algebra simply be at the same time a Banach lattice, an algebra with sub-multiplicative norm and with the product of positive elements being positive. If there is an identity which has norm one we call it a 1-Banach lattice algebra.

At this stage, let me record one important feature of the relationship between the multiplication and order.

Proposition 2.1 In any Banach lattice algebra $A$ (whether real or complex), with multiplication $\star,|x \star y| \leq|x| \star|y|$ for all $x, y \in A$.

In the real case, this is routine. For Banach algebras this was first proved in the complex case by Arendt, [3, Lemma 1.5], whilst a more accessible proof for Archimedean relatively uniformly complete Riesz algebras may be found in Theorem 2 of [16].

We mentioned above that if the identity in a Banach lattice algebra has norm one then it is positive. In fact [7] proves positivity under rather weaker hypotheses. It is worth noting that, whilst a direct converse is not possible, it is modulo a re-norming.

Theorem 2.2 If A is a Banach lattice algebra with multiplication $\star$ and multiplicative identity $e$, the following are equivalent:

(1) $e \geq 0$

(2) There is an equivalent Banach lattice algebra norm on $A,\|\mid \cdot\|$, for which $\|e\|=1$.

Proof We refer the reader to [7] for the proof that (2) implies (1). Assume that (1) holds. As products of positive elements are positive, the map $x \mapsto L_{x}: A \rightarrow \mathcal{L}^{r}(A)$ is positive. Let $\||x|\|=\left\|L_{|x|}\right\|$. As $e$ is positive, $L_{|e|}=L_{e}$ is the identity operator on $A$ so certainly $\|e\| \|=1$. It is clear that $\|\cdot \mid\|$ is a lattice norm as $|x| \leq|y| \Rightarrow L_{|x|} \leq$ $L_{|y|} \Rightarrow\|x\|=\left\|L_{|x|}\right\| \leq\left\|L_{|y|}\right\|=\|y\| \|$. This norm is also sub-multiplicative as if $x, y \in A$ then $|x \star y| \leq|x| \star|y|$ by Proposition 2.1 so that $L_{|x \star y|} \leq L_{|x| \star|y|}=L_{|x|} L_{|y|}$ and hence

$$
\left\|\left|x \star y \left\|\left|=\left\|L_{|x \star y|}\right\| \leq\left\|L_{|x|} L_{|y|}\right\| \leq\left\|L_{|x|}\right\|\left\|L_{|y|}\right\|=\||x\|\mid\| y \| .\right.\right.\right.\right.
$$

It remains only to prove the equivalence of the two norms. As

$$
\left\|L_{|x|} y\right\|=\||x| \star y\| \leq\||x|\|\|y\|=\|x\|\|y\|,
$$

we have $\||x|\|=\left\|L_{|x|}\right\| \leq\|x\|$. On the other hand,

$$
\||x|\|\|e\|=\left\|L_{|x|}\right\|\|e\| \geq\left\|L_{|x|} e\right\|=\||x| \star e\|=\||x|\|=\|x\| .
$$


This may be a suitable occasion on which to ask:

Question 2.3 If a Banach lattice algebra has an approximate identity $\left(e_{\gamma}\right)$ with all $\left\|e_{\gamma}\right\| \leq 1$, must it have an approximate identity composed of positive elements?

We separate our questions into two sections. First we deal with variants of matters that apply to Banach algebras, just modified to take account of the extra order structure that is available here. After that we ask questions that only make sense because of the order structure.

\section{Algebraic considerations}

There are some fundamental constructions in Banach algebra theory that need analogues for Banach lattice algebras.

Completion It is well-known, and routine to prove, that the completion of a normed lattice is a Banach lattice and that the completion of a normed algebra is a Banach algebra, after a possible re-norming to cater for an identity that is introduced by the completion process. It is clear that products of positive elements remain positive in the completion, so the completion of a normed lattice algebra ${ }^{1}$ is certainly a Banach lattice algebra.

Complexification What about complexifying a real Banach lattice algebra? This is vital and is certainly possible in important concrete examples. The norm in the complexification of a real Banach lattice is completely specified by the requirement that $\|z\|=\||z|\|$ and it follows immediately from Proposition 2.1 that the norm in the complexification remains submultiplicative. The complexification process cannot introduce an identity or new positive elements so the complexification of a real Banach lattice algebra is certainly a complex Banach lattice algebra.

Adding an identity Our first uncertainty comes when we try to add an identity. It is not that this is difficult to do, in fact it seems far too easy. Given a non-unital Banach algebra $A$ embedded in a unital Banach algebra $B$, the linear span of $A$ and the identity in $B$ is a unital Banach algebra and (up to a re-norming) independent of the choice of $B$. Nothing similar works for Banach lattices, let alone Banach lattice algebras. There is one rather obvious way of adding an identity to any Banach lattice algebra.

If $A$ is a Banach lattice algebra over $\mathbb{F}$, where either $\mathbb{F}=\mathbb{R}$ or $\mathbb{F}=\mathbb{C}$, then $\mathbb{F} \times A$ is a unital Banach algebra under the multiplication

$$
(\lambda, x) \star(\mu, y)=(\lambda \mu, \lambda y+\mu x+x \star y)
$$

and norm $\|(\lambda, x)\|=|\lambda|+\|x\|$. Defining $(\lambda, x)$ to be positive if $\lambda \geq 0$ and $x \geq 0$ also makes it a Banach lattice. Products of positive elements are positive. This is the only construction that we have come up with which has any claim to being canonical, and we denote it by $A_{e}$. However, in concrete examples, there may be more natural ways of adding an identity.

Example 3.1 Take $A=c_{0}$ with pointwise order and multiplication.

\footnotetext{
1 We have not actually defined these, but the definition should be clear.
} 
(1) $A_{e}$ as defined above is lattice isomorphic to $c_{0}$ (although not isometrically so).

(2) The "obvious" way to add an identity is to embed $A$ in $c$. Although $A_{e}$ and $c$ are isomorphic Banach algebras, they are certainly not isomorphic Banach lattices. For example $A_{e}$ has an order continuous norm but $c$ does not.

(3) Proposition 8.3 of [31] shows that there is a Banach lattice algebra norm on $\mathbb{F}^{2}$ when equipped with the pointwise multiplication and ordered by the positive cone $\{(x, y): x / 2 \geq y \geq 0\}$. In this Banach algebra the identity $(1,1)$ is not positive. Consider the product of this algebra with $c_{0}$ with order specified as the direct sum of the two orders and (commutative) multiplication of $(x, y) \in \mathbb{F}^{2}$ with $\left(z_{n}\right) \in c_{0}$ defined to be

$$
\left(x_{1}, y z_{2}, x z_{3}, y z_{4}, x z_{5}, y z_{6} \ldots\right)
$$

As the positive cone in $\mathbb{F}^{2}$ is contained in the standard one, this extended multiplication still has products of positive elements being positive. Possibly after renorming, this will be a Banach lattice algebra with $(1,1) \oplus(0)$ being the (nonpositive) identity. The linear span of the identity plus the original $c_{0}$ is not a sublattice. This shows that adding an identity need not be reducible to a onedimensional extension and that the added identity need not be positive.

If $B$ is a unital Banach algebra then any algebra homomorphism $T: A \rightarrow B$ extends to unital algebra homomorphism of $A_{e}$ into $B$. We would desire something similar in a Banach lattice algebra setting.

Question 3.2 Is there a canonical way of adding an identity to a Banach lattice algebra $A$ such that if $B$ is a unital Banach lattice algebra then every linear: $A \rightarrow B$ which is both an algebra and lattice homomorphism extends (uniquely) to a unital lattice and algebra homomorphism of this augmented version of $A$ into B?

It will follow from Proposition 3.4 that none of our extensions of $c_{0}$ have this property. The existence of the third example in Example 3.1 shows that the added identity will not in general be positive. That observation prompts:

Question 3.3 Is there a canonical way of adding an identity to a Banach lattice algebra A to produce a 1-unital Banach algebra such that if B is a unital 1-Banach lattice algebra then every linear: $A \rightarrow B$ which is both an algebra and lattice homomorphism extends (uniquely) to a unital lattice and algebra homomorphism of this augmented version of $A$ into $B$ ?

If it exists, such an extension will be quite complicated, even in the 1-Banach lattice algebra case. In particular, we cannot take $A$ to be a lattice ideal in its augmented algebra. In general, what we seem to be looking for seems to be some kind of free unital extension of $A$.

Proposition 3.4 There is no lattice and algebra embedding of $c_{0}$ onto a lattice ideal in a unital Banach lattice algebra A such that, for every 1-Banach lattice algebra B, every lattice and algebra homomorphism $T: c_{0} \rightarrow B$ extends to a unital lattice and algebra homomorphism of $A$ into $B$. 
Proof Let $e$ be the identity in $A$. For the first step, take $B=c$ with the usual order and multiplication and assume that $T: A \rightarrow B$ is a unital lattice and algebra homomorphism extending the identity embedding of $c_{0}$ into $c$. Certainly, $T e=(1)$. If $e_{1}$ is the usual first basic coordinate vector in $c_{0}$ and $|e| \wedge e_{1}=0$ then $0=T|e| \wedge T e_{1}=|T(e)| \wedge e_{1}=(1) \wedge e_{1}=e_{1}$ giving a contradiction. As $c_{0}$ is assumed to be an ideal in $A,|e| \geq \alpha e_{1}$ for some $\alpha>0$.

Now suppose that $S: A \rightarrow\left(c_{0}\right)_{e}$ is a unital lattice and algebra homomorphism extending the identity embedding of $c_{0}$ into $\left(c_{0}\right)_{e}$. As $|e| \geq \alpha e_{1}, S(|e|)=|S(e)| \geq$ $\alpha e_{1}$, which contradicts the fact that $S e$ is disjoint from $e_{1}$.

Apart from the fundamental question of the existence of a categorical unitization, there is also the following question to consider.

Question 3.5 Suppose that $A$ is a non-unital Banach lattice algebra. Let $B_{1}$ and $B_{2}$ be unital Banach lattice algebras, containing $A$ as a sublattice and subalgebra, such that the smallest unital sublattice and subalgebra of $B_{k}$ containing $A$ is the whole of $B_{k}(k=1,2)$. Is it true that for all $x \in A, \sigma_{B_{1}}(a)=\sigma_{B_{2}}(x)$ ? The same question is of interest if $B_{1}$ and $B_{2}$ are restricted to be 1-Banach lattice algebras.

Morphisms, sub-objects and quotients As with studying any any mathematical object, we ought to set Banach lattice algebras in a categorical setting by specifying the morphisms between them. The linear maps that are both algebra and lattice homomorphisms are the obvious ones to work with (and preserving multiplicative identities if they exist), although the restraint of having to preserve both structures will mean that there are relative few examples. Nevertheless, in some important cases there are important examples of such maps. If $X$ and $Y$ are compact Hausdorff spaces and $C(X)$ and $C(Y)$ are given the pointwise lattice and algebra operations, then $T: C(X) \rightarrow C(Y)$ is a unital algebra homomorphism if and only if it maps $\mathbf{1}_{X}$ to $\mathbf{1}_{Y}$ and is a lattice homomorphism. This is likely to be a rather atypical behaviour!

The (closed) kernels of such morphisms will be both algebra and lattice ideals. Again, although these two kinds of (closed) ideals coincide in the $C(X)$ case, in general there will be few such subsets. For example Arendt and Sourour [5] have shown that in $\mathcal{L}^{r}\left(\ell_{2}\right)$ there is precisely one such subset, namely the closure in $\mathcal{L}^{r}\left(\ell_{2}\right)$, for the regular norm, of the finite rank operators. The images of these morphisms will be both subalgebras and sublattices. It is not clear to the author that there is much more to say on this topic. One, possibly rather fanciful, possibility is that certain multiplicatively defined subsets of a Banach lattice algebra, such as the centre, might automatically be sublattices as well.

There is an extensive literature looking at conditions on a unital Banach algebra $A$ such that if $A$ is a unital subalgebra of a larger Banach algebra $B$ then $\sigma_{A}(x)=\sigma_{B}(x)$ for all $x \in A$. These prompt:

Problem 3.6 Find conditions on a unital Banach lattice algebra $A$ such that if $A$ is a sublattice and unital subalgebra of a larger Banach lattice algebra $B$ then $\sigma_{A}(x)=$ $\sigma_{B}(x)$ for all $x \in A$. It would be of particular interest if some familiar order theoretic property of $A$, such as Dedekind completeness, were sufficient. 
Biduals There is a substantial literature on the bidual of a Banach algebra and it is classical (and not very deep) that the bidual of a Banach lattice can naturally be given a Banach lattice structure.

The two Arens multiplications on the bidual of a Banach lattice algebra are certainly Banach algebra multiplications and the method of Theorem 4.1 of [18] shows that they are Riesz algebra multiplications. The first question to ask is whether or not the algebra need be Arens regular, in the sense that both multiplications coincide? Arens gave an example in [6] to show that this is not always true, whilst Buskes and Page [13] show that if $\ell_{1}$ does not embed into the algebra then it must be Arens regular. Of course the multiplication on a Banach lattice algebra may be Arens regular even if it does contain a copy of $\ell_{1}$. For example a Banach $f$-algebra is always Arens regular even if it does contain a copy of $\ell_{1}$, e.g. $\ell_{1}$ with its pointwise multiplication. The same is even true for almost $f$-algebras, [12]. The usual Banach algebra questions, e.g. whether or not the bidual is semi-prime, has an identity, commutativity etc., are relevant and the results in the $f$-algebra setting are of interest. One additional feature of the Banach lattice algebra setting is the existence of the order continuous dual, $E_{n}^{\prime}$ of a Banach lattice $E$. There is a natural embedding of $E$ into $\left(E^{\prime}\right)_{n}^{\prime}$, so all the questions about the bidual, $A^{\prime \prime}$, of a Banach lattice algebra $A$ also apply to $\left(A^{\prime}\right)_{n}^{\prime}$. Many questions may be easier to answer in this setting. Results known in special Riesz algebra settings, such as $[8,17,18,27]$, are certainly of significance in our setting.

Multipliers When $A$ is merely a Banach algebra, the right multiplier algebra of $A$ is the space of bounded linear operators $T: A \rightarrow A$ such that $T(a \star b)=a \star T(b)$ for all $a, b \in A$. In the case of a Banach lattice algebra we should replace bounded by either regular or order bounded. Does this give us a Banach lattice algebra? Hopefully it will not be necessary to assume Dedekind completeness in order to obtain positive results. It is even possible that the order theoretic assumption could be dropped. There are, of course, similar questions to be asked about left multiplier algebras and two-sided multiplier algebras. The motivating example of $C_{0}(\Sigma)$, where the Banach algebra multiplier algebra may be identified with $C^{b}(\Sigma)$, the continuous bounded functions on $\Sigma$, prompts us to pose:

Problem 3.7 Characterise those Banach lattice algebras A for which the multiplier algebra coincides with the ideal centre in the Banach lattice sense, i.e. the space of orthomorphisms on A.

Tensor products A less straightforward project would be to develop a theory of tensor products of Banach lattice algebras. There are well developed theories of tensor products of Banach algebras and of Banach lattices, but they are rather different. One way to tackle tensor products of (sufficiently nice) Banach lattice algebras would be to use the left regular representation of the two factors $A$ and $B$, tensor these as Banach lattices to produce $A \otimes B$ (presumably the Fremlin tensor product), then look at the closed lattice-algebra generated by the induced operators $L_{a} \otimes L_{b}$ for $a \in A$ and $b \in B$. A lot of work would need to be done even if this were possible. However, it will only be in rather special cases that the left regular representation will be sufficiently well-behaved for this approach to work! 


\section{Order theoretic considerations}

Order continuity of multiplication An important existing theory is that of $f$-algebras, where the multiplication is order continuous. Can one develop a general theory of Banach lattice algebras with order continuous multiplication? Will it be much nicer than the general theory? Can we characterize Banach lattice $f$-algebras in some way? Are there other interesting examples? If the norm is order continuous, e.g. $L^{1}(G)$, then multiplication will at least be separately order continuous.

Dedekind completion It is natural to study the Dedekind completion of a Banach lattice, although even that theory is not straightforward.

Question 4.1 Is it possible, in general, to extend the multiplication from a Banach lattice algebra to its Dedekind completion?

On $f$-algebras this is straightforward because of the order continuity of the multiplication and the extension is even unique. Buskes and van Rooij [14] have shown that the extension is possible, although not uniquely, for almost $f$-algebras. Will the assumption of being a Banach lattice algebra, as opposed to a Riesz algebra, help at all?

Classical spaces There are existing results characterizing the classical Banach lattice algebras of the form $C(\Omega)$, dating back to [19], or $L^{1}(G),[22,30]$. What about the most general possible Banach lattice algebra structure on unital AM-spaces or on ALspaces? For example there is a complete description of $d$-algebra multiplications, i.e. Riesz algebra multiplications with $|x \star y|=|x| \star|y|$, on unital AM-spaces, [10,11,22]. It would even be of interest to know what the unital algebra multiplications were.

\section{Representations}

Order left regular representations If $A$ is a Banach lattice algebra then the left regular representation takes values in $\mathcal{L}^{r}(A)$, the algebra of regular operators on $A$. The fact that products of positive elements in $A$ are positive means that if $a \in A_{+}$then $L_{a}$ is a positive operator on $A$. I will (provisionally) call a Banach lattice algebra $A$ order left regular if $a \geq 0 \Leftrightarrow L_{a} \geq 0$. Not all Banach lattice algebras are order left regular, e.g. put the zero multiplication on any Banach lattice then the image of every element will be the zero operator which is certainly a positive operator.

Even if the left regular representation of $A$ is faithful, i.e. injective, it is possible that $A$ is not order left regular. Recall, from Theorem 2.2 of [32], that $\mathcal{L}\left(c_{0}\right)=\mathcal{L}^{r}\left(c_{0}\right)$ and that the operator and regular norms coincide. Let $W$ be the operator on $c_{0}$ with matrix

$$
\left(\begin{array}{cccc}
\frac{1}{2} & \frac{1}{4} & \frac{1}{8} & \ldots \\
0 & \frac{1}{8} & \frac{1}{16} & \ldots \\
0 & 0 & \frac{1}{32} & \ldots \\
\vdots & \vdots & \vdots &
\end{array}\right)
$$


which has norm one. If we define $S \star T=S W T$ on $\mathcal{L}^{r}\left(c_{0}\right)$ then $\|S \star T\|=\|S W T\| \leq$ $\|S\|\|W\|\|T\|=\|S\|\|T\|$, and as $W \geq 0$ we also have $S \star T \geq 0$ if $S, T \geq 0$.

This makes $\mathcal{L}^{r}\left(c_{0}\right)$ a Banach lattice algebra, which will be denoted by $A$. Looking at the action of $W$ on the standard basis vectors, we see that $W$ is not bounded below, so is not invertible and thus $A$ has no identity. It is simple to verify that if $S W=0$ then $S=0$ so that the map $S \mapsto L_{S} \in \mathcal{L}^{r}(A)$ is injective.

If $S$ is the operator represented by the matrix

$$
\left(\begin{array}{ccccc}
1 & -1 & 0 & 0 & \ldots \\
0 & 1 & -1 & 0 & \ldots \\
0 & 0 & 1 & -1 & \ldots \\
\vdots & \vdots & \vdots & \vdots &
\end{array}\right)
$$

then $S W$ is positive so that $L_{S} \in \mathcal{L}^{r}(A)$ is positive even though $S$ is not.

If our Banach lattice algebra $A$ has a positive identity $e$ then matters are much simpler, as then

$$
L_{a} \geq 0 \Rightarrow a=a e=L_{a}(e) \geq 0 .
$$

Lattice left regular representations If $A$ is order left regular then it is both algebra and order isomorphic to its image in $\mathcal{L}^{r}(A)$, but this does not mean that the image is a sublattice. If it is I propose to call A lattice left regular.

Some important examples of Banach lattice algebras $A$ do have the left regular representation being a lattice homomorphism, although it is not trivial to prove that. For example Schep [28] and Synnatzschke [29] have explicitly pointed out that if $E$ is a Dedekind complete Banach lattice and $A=\mathcal{L}^{r}(E)$ then $\left|L_{T}\right|=L_{|T|}$.

For $C(K)$ under the pointwise lattice and algebra operations, the left regular representation maps $C(K)$ onto the ideal centre of $C(K), Z(C(K))$ i.e. the linear operators $T$ on $C(K)$ for which there is $\lambda>0$ with $-\lambda I \leq T \leq \lambda I$. That the left regular representation is a lattice isomorphism is well known, at least it would be if $\mathcal{L}^{r}(C(K))$ were a lattice!

It is routine that if $G$ is a locally compact group then $L^{1}(G)$ is a subalgebra and sublattice of $M(G)$. Thus if $\mu \in M(G)$ and $f \in L^{1}(G)$ then $L_{\mu}(f)=\mu \star f$ makes sense and actually lies in $L^{1}(G)$. In Proposition 3.3 of [4], Arendt showed that the mapping $\mu \mapsto L_{\mu}$ is an isometric lattice and algebra homomorphism of $M(G)$ into $\mathcal{L}^{r}\left(L^{1}(G)\right) .^{2}$ Restricting this map to $L^{1}(G)$ shows that $L^{1}(G)$ is lattice left regular.

Question 5.1 Is $M(G)$ always lattice left regular? If not, find conditions on $G$ under which it is.

In spite of these rather natural cases which are lattice left regular, it is actually rare for the left regular representation to map onto a sublattice. In [31] we showed that for the pointwise multiplication on $\mathbb{R}^{2}$ with positive cone $P_{\alpha}^{1}=\{(x, y): x \geq 0, \alpha x \leq$ $y \leq x\}$, which can be normed to be a unital 1-Banach lattice algebra, the left regular representation is only a lattice isomorphism when $\alpha=-1$.

\footnotetext{
${ }^{2}$ Actually the same is true into $\mathcal{L}^{r}\left(L^{p}(G)\right)$, for $p \in(1, \infty)$ as long as $G$ is amenable.
} 
Problem 5.2 Find an intrinsic characterization of lattice left regular Banach lattice algebras.

Other representations We could, of course, seek maps of a Banach lattice algebra into $\mathcal{L}^{r}(E)$, other than the left regular representation, which preserve both the algebra and lattice operations. Just because the obvious choice doesn't work doesn't mean that no other choice will either. Again, our two dimensional examples show that matters are far from obvious.

For the two dimensional algebra just discussed, if $\alpha=-\frac{1}{n}$ then there is such a representation on $\mathbb{R}^{n+1}$ (and that is the smallest dimension) even when the left regular representation is not a lattice isomorphism. In no other case is there a representation on a finite dimensional space. If $\alpha=0$ there is a representation on an infinite dimensional Dedekind complete Banach lattice but in all other cases, I am unable to show whether or not there is an infinite dimensional representation, although I suspect not.

Given the very subtle differences between these algebras there is no obvious criterion to use which might imply the existence of a nice representation. Of course, we have not definitively answered:

Question 5.3 Is every Banach lattice algebra (isometrically) isomorphic to a closed subalgebra and sublattice of some algebra of regular operators $\mathcal{L}^{r}(E)$ ?

It the answer to that is negative, or if the problem proves difficult, there would be interest in the (possibly) more tractable problem.

Problem 5.4 Find simple conditions on a Banach lattice algebra which guarantee that it is isomorphic to a closed subalgebra and sublattice of some algebra of regular operators $\mathcal{L}^{r}(E)$.

We might have expected closed unital subalgebras and sublattices of $\mathcal{L}^{r}(E)$ to play the same rôle in the theory of Banach lattice algebras as closed subalgebras of the bounded operators do for Banach algebra theory. Whilst I can not quite rule that out, matters are certainly far from obvious. What I certainly do not believe, as some colleagues hope, is that they will play a similar role to $C^{*}$-algebras.

Open Access This article is distributed under the terms of the Creative Commons Attribution 4.0 International License (http://creativecommons.org/licenses/by/4.0/), which permits unrestricted use, distribution, and reproduction in any medium, provided you give appropriate credit to the original author(s) and the source, provide a link to the Creative Commons license, and indicate if changes were made.

\section{References}

1. Abramovich, Y.A., Aliprantis, C.D.: Graduate Studies in Mathematics. An invitation to operator theory, vol. 50. American Mathematical Society, Providence (2002)

2. Allan, G.R.: Introduction to Banach spaces and algebras. In; Oxford Graduate Texts in Mathematics, vol. 20. Oxford University Press, Oxford (2011) (prepared for publication and with a preface by $\mathrm{H}$. Garth Dales)

3. Arendt, W.: Über das Spektrum regulärer Operatoren. Dissertation, University of Tüubingen (1979)

4. Arendt, W.: On the $o$-spectrum of regular operators and the spectrum of measures. Math. Z. 178(2), 271-287 (1981). doi:10.1007/BF01262044 
5. Arendt, W., Sourour, A.R.: Ideals of regular operators on 12. Proc. Am. Math. Soc. 88(1), 93-96 (1983). doi: $10.2307 / 2045116$

6. Arens, R.: The adjoint of a bilinear operation. Proc. Am. Math. Soc. 2, 839-848 (1951)

7. Bernau, S.J., Huijsmans, C.B.: On the positivity of the unit element in a normed lattice ordered algebra. Stud. Math. 97(2), 143-149 (1990)

8. Bernau, S.J., Huijsmans, C.B.: The order bidual of almost $f$-algebras. Trans. Am. Math. Soc. 347(11), 4259-4275 (1995). doi:10.2307/2155037

9. Bonsall, F.F., Duncan, J.: Complete Normed Algebras. Springer, New York (1973) (ergebnisse der mathematik und ihrer grenzgebiete, band 80)

10. Boulabiar, K.: Representation theorems for $d$-rings. Commun. Algebra 32(10), 3955-3967 (2004). doi:10.1081/AGB-200027793

11. Boulabiar, K.: Corrigendum to: Representation theorems for $d$-rings (Commun Algebra 32(10), 39553967, 2004). Commun. Algebra 35(3), 1073 (2007). doi:10.1080/00927870601166206

12. Boulabiar, K., Jabeur, J.: Arens regularity of lattice-ordered rings. Ann. Fac. Sci. Toulouse Math. (6) 19 (2010). (Fascicule special, 25-36, English, with English and French summaries)

13. Buskes, G., Page, R.: A positive note on a counterexample by Arens. Quaest. Math. 28(1), 117-121 (2005). doi:10.2989/16073600509486119

14. Buskes, G., van Rooij, A.: Almost $f$-algebras: structure and the Dedekind completion. Positivity 4(3), 233-243 (2000). doi:10.1023/A:1009874426887 (positivity and its applications, Ankara, 1998)

15. Dales, H.G., Aiena, P., Eschmeier, J., Laursen, K., Willis, G.A.: London Mathematical Society Student Texts. Introduction to Banach algebras, operators, and harmonic analysis, vol. 57. Cambridge University Press, Cambridge (2003)

16. Huijsmans, C.B.: An inequality in complex Riesz algebras. Stud. Sci. Math. Hung. 20(1-4), 29-32 (1985)

17. Huijsmans, C.B.: The order bidual of lattice ordered algebras. II. J. Oper. Theory 22(2), 277-290 (1989)

18. Huijsmans, C.B., de Pagter, B.: The order bidual of lattice ordered algebras. J. Funct. Anal. 59(1), 41-64 (1984). doi:10.1016/0022-1236(84)90052-1

19. Kadison, R.V.: A representation theory for commutative topological algebra. Mem. Am. Math. Soc. 1951(7), 39 (1951)

20. Kantorovich, L.V.: Concerning the general theory of operations in partially ordered spaces. Dok. Akad. Nauk. SSSR 1, 271-274 (1936) (Russian)

21. Kantorovitch, L., Vulich, B.: Sur la représentation des opérations linéaires. Compos. Math. 5, 119-165 (1938) (French)

22. Martignon, L.: Banach lattice algebras with multiplicative modulus and convolution Banach algebras. In: Proceedings of the 12th Brazilian Mathematical Colloquium, vol. I, II (Poços de Caldas, 1979). Conselho Nacional de Desenvolvimento Cientifico e Tecnologico, Instituto de Matematica Pura e Aplicada, Rio de Janeiro, pp. 237-248 (1981)

23. Meyer-Nieberg, P.: Universitext. Banach lattices. Springer, Berlin (1991)

24. Naimark, M.A.: Normed Algebras, 3rd edn. Wolters-Noordhoff Publishing, Groningen (1972) (translated from the second Russian edition by Leo F. Boron; Wolters-Noordhoff series of monographs and textbooks on pure and applied mathematics)

25. Rickart, C.E.: The University Series in Higher Mathematics. General theory of Banach algebras. D. van Nostrand, Princeton (1960)

26. Schaefer, H.H.: Banach Lattices and Positive Operators. Springer, New York (1974) (die grundlehren der mathematischen wissenschaften, band 215)

27. Scheffold, E.: Über Bimorphismen und das Arens-Produkt bei kommutativen D- Banachverbandsalgebren. Rev. Roumaine Math. Pures Appl. 39(3), 259-270 (1994) (German, with English summary)

28. Schep, A.R.: A remark on the uniform zero-two law for positive contractions. Arch. Math. (Basel) 53(5), 493-496 (1989). doi:10.1007/BF01324724

29. Synnatzschke, J.; Über einige verbandstheoretische Eigenschaften der Multiplikation von Operatoren in Vektorverbänden. Math. Nachr. 95, 273-292 (1980). doi:10.1002/mana.19800950125 (German)

30. Taylor, J.L.: Measure algebras. Published for the Conference Board of the Mathematical Sciences by the American Mathematical Society, Providence (1973) (Expository Lectures from the CBMS Regional Conference held at the University of Montana, Missoula 1972, Conference Board of the Mathematical Sciences Regional Conference Series in Mathematics, No. 16) 
31. Wickstead, A.W.: Two dimensional unital Riesz algebras, their representations and norms. Positivity. doi:10.1007/s11117-015-0349-1 (to appear)

32. Xiong, H.Y.: On whether or not $L(E, F)=\operatorname{Lr}(E, F)$. Nederl. Akad. Wetensch. Indag. Math. 46(3), 267- 282 (1984)

33. Zaanen, A.C.: Riesz spaces. II. In: North-Holland Mathematical Library, vol. 30. North-Holland, Amsterdam (1983)

34. Zaanen, A.C.: Introduction to Operator Theory in Riesz Spaces. Springer, Berlin (1997)

35. Zelazko, W.: Banach Algebras. Elsevier, Amsterdam; PWN Polish Scientific, Warsaw (1973) (translated from the Polish by Marcin E. Kuczma) 